\title{
Risk factors for hypertension among adults. An analysis of survey data on chronic non-communicable disease at Gilgel gibe field research center, south west Ethiopia
}

\author{
Tesfa Birlew ${ }^{1,}$, Fessahaye Alemseged ${ }^{2}$ \\ ${ }^{1}$ Department of Public Health, Mizan- Aman Health Science College, Mizan Teferi, south nation nationalities and people regional state \\ (SNNPRS), Ethiopia \\ ${ }^{2}$ Department of Epidemiology, College of public Health and Medical Sciences, Jimma University, Jimma, Oromia regional states, Ethiopia \\ Email address: \\ tesfabirlew@yahoo.com (T. Birlew), fessahayeatd@yahoo.com (F. Alemseged)
}

\section{To cite this article:}

Tesfa Birlew, Fessahaye Alemseged. Risk Factors for Hypertension among Adults. An Analysis of Survey Data on Chronic Non-Communicable Disease at Gilgel Gibe Field Research Center, South West Ethiopia. Science Journal of Public Health. Vol. 3, No. 2, 2015, pp. 281-290. doi: 10.11648/j.sjph.20150302.29

\begin{abstract}
Background: Population based survey study on hypertension risk factors among adults in Ethiopia are lacking. The objective of this study was to assess hypertension risk factors (socio-demographic and behavioural) among adults aged 1564 years, at Gilgel gibe field research center, 2013. Methods: An analysis of population based cross-sectional survey data on chronic non-communicable diseases was carried on, between March and May 15, 2013 at the Field Center. Four thousand \& fifty five individuals (hypertensive=303, non-hypertensive=3752) were involved for analysis. Principal investigator together with data miner extracts the required data. Bivariate analysis of the association between the explanatory and outcome variable were carried out and assessed using Odds ratio with 95\% confidence interval; variables in a binary screening found at p-value $\leq 0.25$ candidate, and then Multiple logistic regression analysis employed to find out significant socio-behavioural factors associated with being hypertensive, employing Statistical Program for Social Science version 20.0. Result: Four thousand and fifty five $(90.7 \%)$ out of the planned sample engross in the analysis (hypertensive $=7.5 \%$; women 179 (4.4\%; Nonhypertensive $=92.5 \%$ ). And the age groups of 45-54 years were substantial number of suffering hypertension $67(7.8 \%)$. Female, able to read and write only, alcohol binge women and vigorous recreational exercise were predictors of hypertension. Men and urban residents were less likely to be hypertensive (Odd Ratio $=0.74$ and, 0.82 , respectively). Current Khat chew was highest among hypertensive (Odd Ratio $=1.07(95 \% \mathrm{CI}$ : 0.84-1.36). Reported risk factor: low fruit \&/ or vegetable serve was the highest both in normotensive (42\%) \& 43\% in hypertensive, and total level of physical inactivity was the lowest among hypertensive (1.7\%), and current alcohol consumption 5.5\% among normotensive. The results of bivariate analysis illustrated female, alcohol binge women and vigorous exercise in leisure were statistically significant with Odd Ratio of (1.35, 6.12 and 2.03) times more likely hypertensive, respectively. Then multivariate analysis, denote the Odd Ratio value of being female was 1.32 , able to read and write only 1.60 , binge women 6.78 and vigorous recreational activity 2.61 times more likely to have the disease, in the current study. Conclusion \& Recommendation: Socio-behavioural risk factors for hypertension were common, so provision of health education \& setting up of and strength a surveillance system for hypertension and its risk factors and further extended studies including biological risk factors; is necessary to reduce the burden of hypertension, in the survey population.
\end{abstract}

Keywords: Risk Factors, Hypertension, Gilgel Gibe Field Research Center, South West Ethiopia

\section{Introduction}

All together chronic non-communicable diseases (CNCDs) were considered a problem of affluent. The epidemiological transition has resulted double burden of communicable diseases \& chronic non-communicable diseases; NCDs [1,2,
9]. According to the dictionary of epidemiology, CNCDs defined as, are non-infectious diseases, of long duration, and chiefly includes cardiovascular diseases (CVDs), cancer, diabetes mellitus (DM) \& chronic respiratory diseases are 
leading causes of death and disability, globally \& in all WHO regions [1, 2, 9]. CVDs a leading causes of NCDs deaths, comprises hypertension / high blood pressure (HTN/HBP) as chronic diseases (CDs) \& proximate biological risk factors for morbidity and mortality worldwide (48\%) and $>80 \%$ in low middle income country (LMIC) and of $34 \%$ NCDs deaths, CVDs accounts $15 \%$ in Ethiopia $[2,6,9]$.

HTN is a silent/ iceberg disease \& it is defined as systolic blood pressure (SBP) persistently $\geq 140 \& /$ or diastolic blood pressure $(\mathrm{DBP}) \geq 90 \mathrm{mmHg}$ or taking anti-hypertensive drug [3]. It is the leading risk factors for CVDs in all WHO regions [3-5, 16, 49], united with other group of diseases it accounts $82 \%$ of CVDs mortality associated to older age, gender, smoking, alcohol consumption, unemployment, low education level, insufficient physical activities, and risky dietary habits $[5,16,8]$. The major identified risk factors for HTN include demographic (sex, age and residence) and Behavioural (tobacco use, alcoholism, khat chewing, low fruit and/ or vegetable intake and physical inactivity) and biological factors [8-9, 11, 19-20]. Studies have indicated that these risk factors are widespread globally [20, 22]. On the other hand it is shown that level of proximate demographic factors: education, occupation and income affect and further influence tobacco use, physical activity and dietary habit $[2,11,12]$. Demographic factors: Age, gender and residence were shown to be associated with HTN [7, 19, 36-38, 51] and Reported factors: smoking, khat chewing, physical inactivity and low fruit and/ or vegetable intake [37, 45] in Ethiopia.

HTN can be prevented if the community gets appropriate information, education and communication on possible risk factors. Most of the risks are attributable to lifestyle and behavioral patterns, and can be changed [11, 16, 20]. Therefore, determining the burden of risk factors for HTN in the population would help to design and implement promotive and preventive measures. In the developing world, wide gap exists between the reality of health burden of HTN and the response to it. If the emergence and prevention of risk factors are left undirected, growth of the problem will continue accelerating and worst $[11-13,20]$. Therefore a balance in prevention and control intervention between the already prevalent infectious diseases and the rising burden of HTN would be the best way forward [13, 14, 20].

In the last few years, life style of the Ethiopian population is changing due to urbanization and demographic transition $[6,10,21,24,54]$. As a result the burden of HTN could be on the rise. In view of the above context and recognizing the rareness of similar studies in the country, this study was conducted to determine risk factors for HTN such as both in proximate and contextual demographic factors and also modifiable behavioural factor: smoking, alcoholism, physical inactivity, suboptimal dietary habit, \& Khat chewing in community setting.

\section{Methods}

This population-based analysis of cross-sectional survey of risk factors for HTN was conducted between March and May, 2013 at Gilgel Gibe Field Research Center (GGFRC) of Jimma University. This study was extension of the survey for determination of magnitude of CNCDs and risk factors of CNCDs for the community at GGFRC [23-24].

For this particular study, a population based cross-sectional survey conducted on a random sample of 5,500 adults aged 15-64 years who are residents of the selected ten kebeles at GGFRC was used, as a data source [23]. Individuals' aged 15 to 64 years from both sexes, who were residents of the 10 kebeles under surveillance by the research center were considered. The sample size was determined based on the original survey [24] and the WHO STEPS guideline [16], which has three steps for stepwise assessment of risk factors for CNCDs. For the current study, Of 4,352 individuals who took part for determination of HTN as risk factors for CNCDs at Step I and Step II, 4,055 adults' aged 15-64 years were involved.

Data collection instruments for the initial survey adapted from WHO Stepwise instrument. Stepwise approach to surveillance (STEPs) is a sequential process for collecting data on CNCDs and their risk factors. It starts with gathering key information on risk factors with a questionnaire, then moves to simple physical measurements and then to more complex collection of blood samples for biochemical analysis. Step 1: contained structured questionnaire composed of sociodemographic variables and questions for assessing behavioural risk factors for CNCDs surveillance. Accompanying with WHO STEPs approach \& employing the initial survey as baseline data: socio-demographic and behavioral variables incur through questionnaire at step I were reviewed to find out possible risk factor for HTN $[16,24]$.

Data collectors of the initial survey study had a minimum of high school completion and competent in Amharic and Oromifa languages. Fifteen interviewers, six physical measurement recorders and three supervisors for CNCDs survey were recruited. Face-to-face interview was conducted after obtained the participant's consent. Three BP readings taken using the WHO recommended automatic BP check. For this particular study, the PI and data miners/extractors reviewed the baseline data, and then required data was extracted, in accordance to WHO steps and the initial survey $[16,24]$.

The initial survey Data were analyzed using SPSS for Windows version 16.0 after double data entry using EpiData version 2, Background of study participants was described and prevalence, and also values for risk factors of CNCDs were classified based on WHO STEPS manual recommendations [23, 24], determined and presented in tables. For the current particular study, baseline data were reviewed; entered data were extracted from those who participated to study HTN as risk factors for CNCDs, and then the data exported into SPSS version 20 for analysis. The PI computed the statistical analysis. The data were processed by using both descriptive summary measures and analytical statistical methods that is frequency distribution, cross tabulation, summary measures (frequency and percentages). 
Bivariate analysis was computed to see the association between the exposure and outcome variables and independent variables had $\mathrm{p}$-value $\leq 0.25$ were candidate to Multiple logistic regression (MLR), employed to find out significant socio-behavioural factors associated with being hypertensive, with Odds ratios (OR) at 95\% confidence intervals (CI) was calculated \& statistical significance were accepted at the 5\% (P-value $<0.05)$, employing with SPSS version 20.0. Risk factors for HTN were determined and organized and presented in tables, graphs and plot form.

The values for risk factors were classified based on the WHO STEPs manual recommendations \& according to the original survey research $[16,23,24]$. Incomplete, distorted and inconsistent data were left out from the analysis. Ethical clearance was obtained from Jimma University's Research and Publication Office, and from the dedicated experts for the field research center; the principal investigator and data extractors only accessing the data maintained confidentiality. Detailed information on methods of the initial survey unexceptionally described in the original articles [23, 24].

\section{Operational Definitions}

Adequate fruit and or vegetable intake: Daily consumption of fruit and/ or vegetable at least five serving (400g= $5 \mathrm{X} 80 \mathrm{gm}$ - a standard portion size is assumed to be $80 \mathrm{~g}[9,47]$.

Current Alcohol Drinker: Reported consumption of alcohol 30 days before the survey.

Behavioral Risk Factors: Risk factors including: smoking, alcohol drinking, dietary habit, khat chewing and level of physical activity

Current khat use: Reported consumption of khat at the time of the survey.

Current smoking: Reported current smoking at the time of the survey.

Hypertension: As mean SBP $\geq 140$ and/ or DBP $\geq 90 \mathrm{mmHg}$, or history of anti-hypertensive treatment.

Low-level of total physical activity: Total physical activity MET $<600$ minutes/week.

Low Serving of Fruits and/ or vegetables: Serving of fruits and/ or vegetables $<5 /$ day.

Modifiable Risk Factors: Those individuals can change to improve health outcomes together with unhealthy diet, physical inactivity, tobacco use, khat chewing and harmful use of alcohol.

Non-modifiable Risk Factors: Characteristics that cannot be changed by an individual / the environment include age and sex.

Not-current alcohol drinker: Alcohol consumption 12 month before the survey excluding current drinker.

Past smoking: Reported earlier/previous history of smoking, but quit at the time of the survey.

\section{Results}

Of the planned sample size of 4,469 adults 15-64 years of age, 4,055 (91\%) were involved with 1937 (47.8\%) male and
$2118(52.2 \%)$ female (female to male ratio of 1.09$)$. Of participated individuals 303 and 3752 were hypertensive and non-hypertensive, respectively.

\subsection{Socio-Demographic Characteristics of Survey Population}

Out of total study participants who reported their educational status, $3137(77.4 \%$; men $=49.6 \%$, women $=$ $50.4 \%$ ) were unable to read and write. Of total survey population who described about their occupation, just about $1741(43 \%)$ were farmers. Nearly $75 \%$ of involved individuals were from rural areas. Sex wise distribution revealed $94(2.23 \%)$ rural males and $142(3.5 \%)$ rural females in hypertensive and $1352(33.3 \%)$ rural males and $1451(35.8 \%)$ rural females in non-hypertensive. About $711(17.5 \%)$ were in the age group of $15-24$ years, 904 (22.5\%) between age of 35-44 years, and age group 25-34 \& 45-54 years were close to 21.1 and $21 \%$, respectively. The participants were all over distributed evenly across in residence.

\subsection{Distribution of Socio-Demographic Risk Factors for HTN, in Survey Population, GGFRC}

Socio-demographic risk factors for HTN included in the analysis were age, sex, residence, occupation, and educational level. Accordingly, 124 (3.1\%) male and 179 (4.4\%) females (dominant cases) were hypertensive, but $3752(92.5 \%)$ were non-hypertensive. Likewise the distribution of cases ranges from $n=55$ (age range of $\leq 34$ and $>54$ years) and $n=67$ (age group of $\geq 35$ and $\leq 54$ years). Correspondingly 78 of males and 121 of female's cases were unable to read \& write. In addition, 141 of hypertensive were farmers. Dominant cases were rural in residential, 237 $(78.2 \%)$.

\subsection{Distribution of Behavioural Risk Factors for HTN, in Survey Population, GGFRC}

Reported risk factors for HTN included in the analysis were cigarette smoking, alcohol drinking, dietary habit, khat chewing and level of physical activity. And about, 303(7.5\%) were hypertensive. More than 5\% male and $4.5 \%$ female were smokers (hypertensive $=22$ and non-hypertensive $=363$ ). Also 226(6.0\%) non-hypertensive and 20(6.6\%) hypertensive were past daily smokers. Alcohol consumption status of nonhypertensive $=3752$, and hypertensive $=303$ ) were $12.7 \%$ and $12.5 \%$. Over and above $42 \%$ of the population; nonhypertensive $(38.8 \%)$ \& hypertensive $(3.2 \%)$ ate $<$ five servings of fruit and/ or vegetables a day ( 0 serving $(<1 /$ day) $=92.4$ and $7.6 \%, 1-4$ serving $=92.5$ and $7.5 \%$ ), respectively. Low level of total physical activity was found in 561(15\%) and in 52(17.2\%) of non-hypertensive and hypertensive individuals, respectively. Concerning to khat chewing $1462(39 \%)$ and $123(40.6 \%)$ of normotensive and counterparts were current chewer. 


\section{Risk Factors for Hypertension}

\subsection{Socio-Demographic Risk Factors}

Binary logistic regression was computed to assess the association between socio-economic status and being hypertensive. Age-specific distribution of hypertensive depicted that the prevalence of HTN rises steeply with increase in age. Maximum increase in the frequency of HTN i.e. $90 \%$ is observed from age group $35-44$ to $\geq 45-54$ years. Female individuals were 1.35 times more likely to be hypertensive than male and statically significant. Those who were age group of $45-54$ were 1.02 times more likely to be hypertensive than age group of 15-24 years. Concerning to educational level: able to read and write only were 1.57 times more likely to be hypertensive as compared to those unable to read and write \& statistically significant. Other risk sociodemographic factors were rural residence $(\mathrm{OR}=1.2(95 \% \mathrm{CI}$ : 0.92-1.61), occupation: daily laborer, $\mathrm{OR}=1.30(95 \% \mathrm{CI}=$ $0.70-2.42)$, unpaid employee $1.42(95 \% \mathrm{CI}=0.42,4.77)$ and home maid ( 1 out of 25 was cases) with $\mathrm{OR}=0.47(0.06-3.52)$.

\subsection{Behavioural or Reported Risk Factors for HTN}

Binary logistic regression was workout to describe the association among reported risk factors $\&$ being hypertensive. Individuals who were current smoker were 1.09 times more likely to be hypertensive than non-smokers, similarly those currently smoke (1-20 cigarettes per day) or light smokers were 1.36 times more likely hypertensive as compared to the non-user; however these were not statistically significant. Whereas female drank $\geq 4$ drinks during a single occasion were $6.01(\mathrm{OR}=6.12(95 \% \mathrm{CI}: 4.12-9.20)$ times more likely hypertensive (P-value $\leq 0.001)$. In addition, past daily smokers were 1.10 times to be hypertensive as compared to counterparts. Alcohol consumption of (1-6 times /week) among not current drinker and current drinker were 1.20 and 1.01 times more likely being hypertensive, correspondingly. Zero serving ( $<1$ per day) of fruit and/ or vegetable was 1.80 times being hypertensive as compared to $1-4$ serving per day $(\mathrm{OR}=1.76)$.

Those who were participate in: vigorous recreational exercise for at least 10 minute were 2.03 times more likely to develop HTN and significant, moderate recreational activity 1.43 times more likely and suggestively significant ( $\mathrm{P}$-value $=0.078$ ). However, total level of physical activity showed that those who were physically inactive (low) were 1.19 times more likely hypertensive and moderately active $(\mathrm{OR}=1.18)$. People who were ever and currently chew khat were 1.067 and 1.07 times more likely to be hypertensive compare to counter parts, in turn, on the other hand insignificant $(\mathrm{P}>0.05)$.

\subsection{Multivariate Analysis for Factors Independently Associated with HTN}

For multivariate logistic regression 23 variables include: socio-demographic characteristics and behavioural condition have $p$-value of $\leq 0.25$ were candidate. And then gender: female $(\mathrm{OR}=1.32)$, educational level(able to read and write only, $\mathrm{OR}=1.60$ ), alcohol use (binge women, $\mathrm{OR}=6.78$ ) \& participated in vigorous recreational activity $(\mathrm{OR}=2.61)$ were predictors.

\section{Discussion}

Risk factors distribution assessment is essential for prevention and control of CVDs, a major CNCDs holding HTN as single risk factors. This study focused on finding out socio-behavioral risk factors for HTN. However, underreporting of behavioural risk factors for HTN could have happened due to issues of sensitivity and self-reported data (social desirability bias). This survey data analysis is the first of its kind in the survey population of finding out risk factors for HTN in a community setting, in particular. Of 4,469 planed sample 4,055(91\%) were included in the analysis. Slight difference in sex, age and residence based distribution of hypertensive cases perhaps affected the true distribution of the risk factors among those individuals and results should be interpreted with cautions.

The broad mechanisms involved in the development of HTN include biological and reported risk factors [2, 6, 2526]. By proxy HTN considered as single leading cause for CNCDs, particularly for CVDs, but in LMIC data on factors associated with it still scarce [7, 9, 35]. Of total of 4,055 individuals included in the analysis, $7.5 \%$ (reported $=2.5 \%$ and observed $=5 \%$ ) were hypertensive (largely rural females) which is close to the number of cases in the initial survey [23, 24]. Like other CDs, HTN is highly driven by sociodemographic and behavioural related risk factors [5, 11-14, $16,36]$.

Socio-demographic risk factors: Social determinants should not only juxtapose with traditional risk factors acting disease together. Taking a dynamic perspective on these social determinants of health, and in particular viewing them in a biological and epidemiological context, emphasizes the fact that intervention as early in life as possible is desirable in order to prevent hypertensive disease. In the current study SES included in the analysis were age, sex, educational level, residence and occupation.

Sex: Many reports, and review articles described HTN is highly distributed in LMIC \& people are affected at much younger ages (mainly in men than women) in developing than developed countries $[2,5,22]$. Of total non-hypertensive and hypertensive, $1939(51.7 \%)$ and $179(59.1 \%)$ were females, moreover $6.4 \%$ and $8.5 \%$ were hypertensive males and females, respectively. All around female was found to be significance both in bivariate and in multivariate analysis. And females were 1.32 times more likely to be hypertensive, and was predictors, this finding is consistent with findings reported by the side of $[30,33]$ and other reports where HTN mainly affected females at advanced age than males (suffer before middle and at young age, but disagree with the study done at $[29,36,44]$ and across in three population in Africa and Asia (Ethiopia, Vietnam and Indonesia) [22].

Age: Studies agree with the fact that HTN increased with 
age, due to vascular change and atherosclerosis. In the current finding age wise distribution of hypertensive was increase from age $25-34(6.9 \%)$, followed by age group of 35 44 years $(7.4 \%)$, and then $45-54(7.8 \%)$ were more likely hypertensive as compared to age 15-24 having an OR $(0.88$, $0.96,1.02)$, but the result is not unexpected in light of a study done on [36], as age increase there were high odds among males, but females. In contrast to the current result, in all (Ethiopia, Vietnam and Indonesia) except Hawaii [49] studies age was significantly associated with HTN, possibly may be difference in age group of various study, in most studies age distribution range from 15-100 years. The result implied as compared to developed country, aged women(above middle and at old age) affected more than men(mainly suffer at young) in developing country $[2,5,22]$, suggested that widen age range may be help to distinguish the disagreement/ significant change.

Residence: Urbanization and globalization leads to an age old disease. The urban to rural gradient in HTN is wider in some countries than others. However, the gap between wealthier and poorer countries, as well as urban and rural populations is narrowing rapidly. The prevalence of HTN in urban and rural populations is comparable. Thus, the public health importance of HTN is apparent in both urban and rural populations in Africa including Ethiopia [7, 22]. HTN was more likely in rural than urban $7.8 \%(\mathrm{OR}=1.2)$, this is possibly due to the highest distribution of socio-behavioural risk factors in rural areas, but statistically insignificant, and consistent finding with study conducted at [7, 19] as well study across in three population of Africa and Asia, but slight difference among urban residents of Ethiopia $(\mathrm{OR}=1.02)[7$, 22]. Difference in risk factors in various studies observed may be because of variation in gender wise distribution, and geographical differences, many conducted at urban residents, implied that risk factors and risks are across in residence, and consider both in and semi-residence is important for screening and prevention. .

Education: Higher level of education is associated with greater awareness regarding health and diseases. Multiple reports and studies [2, 7, 20,49] have shown that education significantly reduces the prevalence of illness (educated were less to be risk than less educated or uneducated). Some studies find that education level is inversely associated with HTN, resemble as a study conducted on Punjabi females; literates are more hypertensive than illiterates [30] while others suggest that there is a direct proportion between them. The present sample is in conformity with the latter studies in India; where less educated and uneducated were more likely hypertensive than educated. The result of the current study exhibited also the theoretical aspect, most hypertensive cases were unable to read and write 199(7.0\%). The finding revealed hypertensive case were decrease as their educational level increase namely from $10.6 \%$ to $8.1 \%$ and then $5.5 \%$, OR $(1.57,1.17,0.77)$ from able to read and write followed by 1-4 grade, and finally those attend $>5$ grade, respectively. Participants able to read and write only were 1.60 times more likely to be hypertensive than those unable to read and write and statistically significant while in contrast with a study done in eastern Uganda, and India whereas attended tertiary educations and educated were more hypertensive [30, 34], on the other hand there were no significant difference among three population in Africa and Asia [22].

Occupation: With modernization and changing demographic also known as SES profile of the population, HTN are assuming an increasing importance. In the current study the odds of being hypertensive among government employee, merchant, daily laborer and unpaid worker were markedly increase OR $(1.10,1.16,1.30$, and 1.42) times more likely hypertensive as compared to farmers, respectively, possibly due to stress, and lack of incentive, but statistically insignificant, may be due to scattered of case across factors, and conflicts with study reported at Turkish [29].

Behavioural risk factors: $\mathrm{HTN}$ is highly driven by reported risk factors. During the initial population based survey, the distribution of these factors were (95\%), current khat chew and alcohol use was the highest and lowest $(38.6 \%$ and $7.1 \%$ ), in rural men $(67.3 \%)$ and rural women $(2.0 \%)$, definitely [23, 24]. Nonetheless, in the current study; low serving of fruit and/ or vegetable was highest in the survey population, both in normotensive $(38.8 \%)$ and in hypertensive (3.2\%). However, alcohol use before 30 days of the survey was the lowest in normotensive and hypertensive groups $(5.1 \%$ and $0.004 \%)$, yet $5.3 \%$ within hypertensive individuals.

Current tobacco smoking: The use or misuse of addictive substances, such as cigarettes, is increasingly prevalent in Ethiopia. In the current study 31(10\%) and 354(9.4\%) were current smoking as compared hypertensive with normotensive, highest both in rural $26(8.6 \%)$ and $265(7.1 \%)$, and among rural men 14(4.6\%) and rural female135 (3.6\%), respectively. In addition, current smoking ranks as the second behavioural risk factors among hypertensive and highly distributed as compared to normotensive. Smokers were 1.09 times more likely to have the condition, so far no significance was found, the same finding was met in the study of Saudi Arabia [50]. The distribution is comparable study conducted in Ethiopia [22, 37].

Alcohol Consumption: The relationship between HTN and alcohol drink is unclear, but many reports and studies [43, 44, 53] suggested among drinkers; not binge drinkers there appears to be J/or U shaped relation, and heavy use was associated with HTN. And among young women who use light to moderate alcohol reduce the risk of developing hypertensive $\mathrm{CDs}$ and show $\mathrm{J}$ relationship. The finding revealed alcohol consumption was higher for rural than urban and among females than men population and $16(5.3 \%)$ and $207(5.5 \%)$ were current alcohol use, highest both in rural $14(4.6 \%)$ and 161(4.3\%), and among rural women 9(3\%) and $83(2.2 \%)$, amongst hypertensive and normotensive, separately. Similarly, number of days drinking alcohol and duration has high odds to have the disease as compared to abstainers OR (1.40, 1.01), congruently. This finding is consistent with the study conduct at Addis Ababa [22]. 
Though, binge women were 6.12 and 6.78 times more likely to have the condition as compared to non-binge both in biand multivariate analysis and statistically significant, consistent with the study conducted at [53], where binge men was less likely $(\mathrm{OR}=0.074)[22,43,48]$ or women were 13 times being hypertensive. Suggested that abstainers are at risk as compared to light to moderate user, and conversely heavy drank lead to adverse effect (HTN).

Fruit and/ or vegetable intake: It is widely accepted that fruit and/ or vegetable are important component of healthy diet and that their consumption could help prevent a wide range of CVDs including hypertensive CDs and WHO aim to promote an increases in consumption of it. More than $42 \%$ of the survey population consume fruits and/ or vegetables below adequate level ( $<$ five servings/day), is lower than WHO, 2003 report [54]. One possible reason contributing for such a difference could be the fact that coverage of the survey population. Low fruit and/ or vegetable consumption is an important risk factor for CDs, but for many (mainly developing) countries, no prevalence data have ever been published. As compared hypertensive with normotensive, $130(43 \%)$ and $1574(42 \%)$ were serving $<5$, highest both in rural $99(32.7 \%)$ and $1159(30.9 \%)$, and among rural men 91(30\%) and rural women 835 (22.3\%), consistently [36, 44].

Physical activity: Participate at work, recreational or leisure's reduced the burden of hypertensive CDs, particularly among young men and above middle aged (older women) since the occurrence of HTN varies across gender. In the current study $5(1.7 \%)$ and $561(15 \%)$ were physically inactive, highest both in rural 44(14.5\%) and 376(10\%), and among rural women 28(9\%) and rural men 192 (5.1\%), compared hypertensive to normotensive, correspondingly. Concerning to the total level of physical activity, the odds of being hypertensive was increased from moderately active to those physically inactive ( $\mathrm{OR}=1.187$ and 1.191$)$, respectively. However, it was not found to be significance, but as compared those participated in vigorous leisure for at least 10 minute were 2.61 times more likely to develop the condition, and significant. The possible reason for this strong association may be that, in women the possible preventive effect of leisure physical activity occurs in later life (older age) as a result of later appearance of HTN in women, since majority of case were women found at middle age), the opposite is true in case of men [36, 41, 42, 44] and consistent finding with a study done among Turkish adults: Trabzon and Saudi Arabia[29, 41], contrasted with what Goma reported at Zambia [36].

Khat chewing: Is the most important highly distributed reported factors in the population (39.1\%), but as compared those hypertensive and normotensive users, in the current study $123(40.6 \%)$ and $1462(39 \%)$ were current khat chew, highest both in rural 94(31\%) and 1095(29.2\%), and among rural men 52(17.2\%) and rural female 552 (14.7\%), congruently. Besides of the above reported risk factors those who use khat (past daily and current chew), the odd of developing the condition was 1.70 , conversely no significant difference. The finding was consistent with the study conducted at $[22,37,38,45]$, however conflict with study conducted at Saudi Arabia as khat user were more likely hypertensive and depicted a significance difference [50].

Table 1. Bivariate logistic regression for socio-demographic risk factors associated with HTN, GGFRC, 2013.

\begin{tabular}{|c|c|c|c|c|c|c|}
\hline \multirow{2}{*}{\multicolumn{2}{|c|}{ Socio-demographic characteristics }} & \multicolumn{2}{|c|}{ Hypertensive } & \multicolumn{2}{|c|}{ Non-hypertensive } & \multirow{2}{*}{$\begin{array}{l}\text { Crude } \\
\text { OR (CI: 95\%) }\end{array}$} \\
\hline & & No & $\%$ & No & $\%$ & \\
\hline \multirow{3}{*}{ Sex } & Male & 124 & 6.4 & 1813 & 93.6 & 1.00 \\
\hline & Female & 179 & 8.5 & 1939 & 91.5 & $1.35(1.06-1.71)$ \\
\hline & $15-24$ & 55 & 7.7 & 656 & 92.3 & 1.00 \\
\hline \multirow{4}{*}{ Age in years } & $25-34$ & 59 & 6.9 & 798 & 93.1 & $0.88(0.60-1.29)$ \\
\hline & $35-44$ & 67 & 7.4 & 837 & 92.6 & $0.96(0.66-1.38)$ \\
\hline & $45-54$ & 67 & 7.8 & 787 & 92.2 & $1.02(0.70-1.47)$ \\
\hline & $55-64$ & 55 & 7.5 & 674 & 92.5 & $0.97(0.66-1.44)$ \\
\hline \multirow{3}{*}{ Residence } & Urban & 66 & 6.5 & 949 & 93.5 & 1.00 \\
\hline & Rural & 237 & 7.8 & 2803 & 92.2 & $1.2(0.92-1.61)$ \\
\hline & Unable R\&W1 & 199 & 7.0 & 2646 & 93.0 & 1.00 \\
\hline \multirow{4}{*}{ Educational Level } & Able to R\&W only & 64 & 10.6 & 541 & 89.4 & $1.57(1.17-2.12)$ \\
\hline & 1-4 grade & 21 & 8.1 & 239 & 91.9 & $1.17(0.73-1.87)$ \\
\hline & $5-8$ grade & 12 & 5.5 & 208 & 94.5 & $0.77(0.42-1.40)$ \\
\hline & $\geq 9$ grade & 7 & 5.6 & 118 & 94.4 & $0.79(0.36-1.71)$ \\
\hline \multirow{10}{*}{ Occupation } & Farmer & 141 & 8.1 & 1600 & 91.9 & 1.00 \\
\hline & Gov't employee & 11 & 9.2 & 108 & 90.8 & $1.16(0.61-2.20$ \\
\hline & Merchant & 17 & 8.8 & 176 & 91.2 & $1.10(0.65-1.86)$ \\
\hline & Daily laborer & 12 & 10.3 & 105 & 89.7 & $1.30(0.70-2.42)$ \\
\hline & Unpaid & 3 & 11.1 & 24 & 88.9 & $1.42(0.42-4.77)$ \\
\hline & Student & 13 & 5.9 & 208 & 94.1 & $0.71(0.40-1.28)$ \\
\hline & Housewife & 47 & 6.6 & 665 & 93.4 & $0.80(0.57-1.13)$ \\
\hline & Pensioner & 4 & 7.8 & 47 & 92.2 & $1.0(0.34-2.72)$ \\
\hline & Unemployed & 2 & 3.0 & 65 & 97.0 & $0.35(0.09-1.44)$ \\
\hline & Other & 52 & 7.0 & 695 & 93.0 & $0.85(0.61-1.20)$ \\
\hline
\end{tabular}

1 R \& W- Read and Write only 

Chronic Non-Communicable Disease at Gilgel Gibe Field Research Center, South West Ethiopia

Table 2. Bivariate logistic regression for behavioural-related factors associated with HTN in adults, GGFRC, 2013.

\begin{tabular}{|c|c|c|c|c|c|c|}
\hline \multirow{2}{*}{ Behavioural Risk Factors } & & \multicolumn{2}{|c|}{ Hypertensive } & \multicolumn{2}{|c|}{ Non-hypertensive } & \multirow{2}{*}{$\begin{array}{l}\text { Crude } \\
\text { OR (CI: 95\%) }\end{array}$} \\
\hline & & No & $\%$ & No & $\%$ & \\
\hline \multirow{2}{*}{ Current smoker } & No & 272 & 7.4 & 3398 & 92.6 & 1.00 \\
\hline & Yes & 31 & 8.1 & 354 & 91.9 & $1.09(0.74-1.61)$ \\
\hline Current daily smokers & Yes & 25 & 7.2 & 321 & 92.8 & $0.96(0.63-1.47)$ \\
\hline \multirow{2}{*}{ Cigarette consumed/day } & Non-smoker & 252 & 6.7 & 3500 & 93.3 & 1.00 \\
\hline & Light smoker & 27 & 8.9 & 279 & 91.1 & $1.36(0.90-2.06)$ \\
\hline \multirow{2}{*}{ Past daily smoker } & No & 283 & 7.4 & 3526 & 92.6 & 1.00 \\
\hline & Yes & 20 & 8.1 & 226 & 91.9 & $1.10(0.69-1.77)$ \\
\hline \multirow{2}{*}{ Drink alcohol in the last 12 months } & No & 282 & 7.5 & 3486 & 92.5 & 1.00 \\
\hline & Yes & 21 & 7.3 & 266 & 92.7 & $0.98(0.62-1.55)$ \\
\hline \multirow{3}{*}{$\begin{array}{l}\text { Freq. of drinking alcohol in the last } \\
12 \text { month. }\end{array}$} & Abstainer & 281 & 7.4 & 3537 & 92.6 & 1.00 \\
\hline & 1-6 times a week & 11 & 8.7 & 116 & 91.3 & $1.40(0.64-2.24)$ \\
\hline & 1-3 days/mos. & 11 & 10.0 & 99 & 90.0 & $1.20(0.74-2.64)$ \\
\hline Drink alcohol in the last 30 days & No & 286 & 7.5 & 3543 & 92.5 & 1.00 \\
\hline \multirow{2}{*}{ Alcohol consumption status } & Abstainer & 284 & 7.5 & 3484 & 92.5 & 1.00 \\
\hline & Ever drinker & 19 & 6.6 & 268 & 93.4 & $0.87(0.54-1.41)$ \\
\hline \multirow{2}{*}{ Alcohol Binge women } & $<4$ drinks & 264 & 6.7 & 3664 & 93.3 & 1.00 \\
\hline & $\geq$ drinks occasion & 39 & 30.7 & 88 & 69.3 & $6.12(4.12-9.20)$ \\
\hline \multirow{3}{*}{ Fruit and or vegetable /serving } & Low serving $<5$ & 130 & 7.3 & 1574 & 92.7 & $1.04(0.82-1.32)$ \\
\hline & Adequate & 173 & 10.2 & 2178 & 89.8 & 1.00 \\
\hline & 0, serving $<1$ & 130 & 7.6 & 1574 & 92.4 & $1.80(0.65-4.97)$ \\
\hline \multirow[t]{2}{*}{ Fruit \& or vegetable serving/ day } & 1-4 serving & 169 & 7.5 & 2091 & 92.5 & $1.76(0.64-4.85)$ \\
\hline & $\geq 5$ serving & 4 & 4.4 & 87 & 95.6 & 1.00 \\
\hline \multirow{2}{*}{$\mathrm{VWE}^{2}$} & No & 161 & 7.1 & 2109 & 92.9 & $0.88(0.70-1.12)$ \\
\hline & Yes & 142 & 8.0 & 1643 & 92.0 & 1.00 \\
\hline \multirow{2}{*}{$\mathrm{MWE}^{3}$} & No & 78 & 7.4 & 975 & 92.6 & $0.99(.76-1.29)$ \\
\hline & Yes & 225 & 7.5 & 2777 & 92.5 & 1.00 \\
\hline \multirow{2}{*}{ Walking/cycling/ } & No & 18 & 6.5 & 261 & 93.5 & $0.85(0.52-1.38)$ \\
\hline & Yes & 285 & 7.5 & 3491 & 92.5 & 1.00 \\
\hline \multirow{2}{*}{$\mathrm{MRE}^{5}$} & No & 273 & 7.3 & 3484 & 92.7 & 1.00 \\
\hline & Yes & 30 & 10.1 & 268 & 89.9 & $1.43(0.96-2.13)$ \\
\hline \multirow{3}{*}{ TLPA $^{6}$ (MET-Minute/week) } & High: $(\geq 1500)$ & 234 & 7.2 & 3007 & 92.8 & 1.00 \\
\hline & Moderate: (600 -1499) & 17 & 8.5 & 184 & 91.5 & $1.19(0.71-1.99)$ \\
\hline & Low: $(<600)$ & 52 & 8.5 & 561 & 91.5 & $1.191(0.87-1.63)$ \\
\hline \multirow{2}{*}{ Past khat chew } & No & 172 & 7.3 & 2189 & 92.7 & 1.00 \\
\hline & Yes & 131 & 7.7 & 1563 & 92.3 & $1.07(0.84-1.35)$ \\
\hline \multirow{2}{*}{ Current khat chew } & No & 180 & 7.3 & 2290 & 92.7 & 1.00 \\
\hline & Yes & 123 & 40.6 & 1462 & 39.0 & $1.07(0.84-1.36)$ \\
\hline
\end{tabular}

Table 3. Multivariate logistic regression for socio-behavioural factors associated with HTN, GGFRC, 2013.

\begin{tabular}{|c|c|c|c|c|}
\hline Predictor Variables & & Hypertensive No (\%) & Non-hypertensive No (\%) & Adjusted OR(95\% CI) \\
\hline \multirow{2}{*}{ Sex } & Male & $124(6.4)$ & $1813(93.6)$ & 1.00 \\
\hline & Female & $179(8.5)$ & 1939(91.5) & $1.32(1.04-1.68)$ \\
\hline \multirow{2}{*}{ Residence } & Urban & $66(6.5)$ & $949(93.5)$ & 1.00 \\
\hline & Rural & $237(7.8)$ & $2803(92.2)$ & $1.17(0.88-1.57)$ \\
\hline \multirow{2}{*}{ Educational Level } & Able to read and write only & $64(10.6)$ & $541(89.4)$ & $1.60(1.19-2.15)$ \\
\hline & Other & $239(6.9)$ & $3211(93.1)$ & 1.00 \\
\hline Cigarette consumed/day & Non-smoker & $276(7.3)$ & $3500(92.7)$ & $1.47(0.96-2.25)$ \\
\hline Alcohol & $<4$ drinks in single occasion & $264(6.7)$ & $3664(93.3)$ & 1.00 \\
\hline Binge women & $\geq 4$ drinks in single occasion & $39(30.7)$ & $88(69.3)$ & $6.78(4.53-10.16)$ \\
\hline \multirow{2}{*}{ Physical activity } & Vigorous recreational activity & $34(13.4)$ & $220(86.6)$ & $2.61(1.47-4.63)$ \\
\hline & No & $269(7.1)$ & $3532(92.9)$ & 1.00 \\
\hline
\end{tabular}

$2 \mathrm{VWE}=$ Vigorous work exercise

3 MWE=Moderate " "

$4 \mathrm{VRE}=$ Vigorous recreational exercise

5 MRE=Moderate" "

6 TLPA $=$ Total level of physical activity $($ MET=Metabolic equivalent $)$ 


\section{Conclusion and Recommendation}

\subsection{Conclusion}

In conclusion the distribution of risk factors for CNCDs is considerably high in the study population. Among hypertensive and normotensive the highest and lowest reported risk factors were khat chew $(40.6 \%)$ and low fruit and/ or vegetable serve $(7.3 \%)$ and among counter parts low fruit and/ or vegetable serve (92.7\%) and khat chew (39\%). In terms of residence alcohol consumption, low dietary intake of fruits and/ or vegetables, and low level of physical activity, smoking and khat chew habits were more common in rural area, among hypertensive women in the first three and among men in the last two behavioural factors, congruently.

These findings are crucial for evidence based decision making. It will help policy makers for planning of preventive and control measures of these modifiable risk factors. This study will also give baseline information that will enable researchers to conduct an extended inclusion of biological and biochemical risk factors and follow-up studies. There were associations between socio-demographic factors (being female and low education level) along with behavioural factors: binge alcohol use, and vigorous leisure recreational activity for at least 10 minute), in the survey population, GGFRC, Jimma zone. And there was no an association between fruit and/ or vegetable serving, and total level of physical activity with HTN. In spite of this, low fruit \&/ or vegetable serve, was common, followed by khat chew, then physical inactivity, go along with tobacco smoking and lastly alcohol use. In addition, to the assessed, involving other biophysical and chemical risk factors will be helped in understand significance difference with the risk.

\subsection{Recommendation}

The findings confirm the growing public health challenge of HTN and associated factors as a public health problem in the survey population in particular. Based on the findings of the study the following proposal are forwarded (for all stake holders' including. Zonal, federal health office as well woreda administrative):

- More community-based studies of this nature are required to determine the extent of this problem, and the risk factors for HTN in the communities.

- Through involving biophysical and chemical factors together with the assessed factors will be helped to find out the significant difference with the risk.

- Interventions aiming at reducing the risk factors identified in this study are needed, and should target both the high risk and the overall population in order reduces the risk and prevalence of HTN. The prevalence was higher in females than males and increased with age.

- $\quad$ Educating the people on the consequence and associated factors could be done in form of campaigns at work places educating people on HTN, the importance of screening for HTN and the association between HTN with risk factors.

- $\quad$ Set up and strengthening a surveillance system for risk factors of HTN which will be used to monitor and evaluate health education and promotion activities. Expand Information on risk factors; this may serve as a system to monitor the prevalence of HTN and risk factors and used to evaluate interventions put in place.

\section{Competing interests}

The authors announce that they have no conflict of interests.

\section{Authors' contributions}

TB (Ethiopia); Involved in the conception, design, analysis, interpretation, report writing and manuscript writing.

FA (Ethiopia); Contributed to the design of the study.

Authors read and approved the final manuscript version submitted for publication.

\section{Acknowledgement}

We extend our extremely grateful appreciation to data extractors or miners and dedicated experts of the field research center; who encouraged us to conduct this recently hot issue on prevention and control of CNCDs including HTN at GGFRC \& delivering all relevant materials that were helped to us during work. The study was funded by Jimma University, College of Public Health and Medical Science.

\section{References}

[1] Prevett M. Chronic non-communicable diseases in Ethiopia. A hidden burden. Ethiop J Health Sci.2008; 22(2):1-2.

[2] WHO. Global status report on no-communicable diseases. 2010:1-160.

[3] Bakris GL, Black HR, Cushman WC, Green LA, Izzo JL, Jones DW, et al. The seventh report of the joint national committee on prevention, detection, evaluation, and treatment. Clinician's corner.2003; 289(19):2560-2572.

[4] Margaret McDonald, Françoise Pickart M. The global burden of no-communicable diseases. 2009:1-16.

[5] Mocumbi AOH, Ferreira MB. Neglected cardiovascular diseases in Africa. Challenges and Opportunities. JACC.2010; 55(7):680-7.

[6] Federal Ministry of Health in Ethiopia (FMOH). The health Sector development programmefour (IV) in Ethiopia. 2010:157.

[7] Tesfaye F. Epidemiology of cardiovascular risk factors in Ethiopia: The rural-urban gradient. Sweden: Umea University, 2008:1-76.

[8] World Economic Forum (WEF). The global economic burden of NCDs. 2011:1-35. 
[9] WHO. Non-communicable diseases country profiles. 2011:519.

[10] ICAP. Strengthening chronic disease services in Ethiopia Lessons Learned from HIV / AIDS program implementation. 2010:11-17.

[11] Chockalingam A, Facc MS, C NRCF, C JGFF. Worldwide epidemic of hypertension. 2006; 22(7):553-5.

[12] AjeA, Adebiyi A and FalaseAO. Hypertensive heart disease in Africa. SA heart. 2009; 6(1):42-51

[13] Marleen E. Hendriks, Ferdinand W. N. M. Wit, Marijke T. L. Roosetal. Hypertension in Sub-Saharan Africa.Cross-sectional surveys in four rural and urban communities. 2012; 7 (3): 1-10

[14] Res M, Jaipur T, VI J. Hypertension \& pre-hypertension in developing countries. 2008:688-90.

[15] Tuso PJ. Serve Ethiopia. The permanent journal/ summer 2009/.2009, 13(3):61-4.

[16] WHO. Rationale for surveillance of chronic disease risk factors .WHO steps surveillance. 2008: 1-17. Available at: www.who.int/chp/steps/resources/database/en/index.html

[17] Solomon T, Mariam MH, Selassie H. Ethiopia Country Report.RAD AID org. 2007:1-25.

[18] The Harvard school of public health multidisciplinary international research training program, Addis continental institute of public health and international clinical laboratories. Symposium on non-communicable diseases in Ethiopia. 2012:1-3

[19] Tesfaye F, Nawi NG, Minh HV, Byass P, Berhane Y, Bonita R, et al. Association between body mass index and blood pressure across three populations in Africa and Asia. $\mathrm{J}$ of Human Hypertension. 2008; 21:28-37.

[20] WHO. New data highlight increases in hypertension, diabetes incidence: Geneva, 2012:1-87

[21] Central Statistical Agency (CSA) [Ethiopia]. Ethiopia demographic and health survey. Central Statistics Authority, Addis Ababa. 2011: 1-22.

[22] Tesfaye F, Byass P, Berhane Y, Bonita R, Wall S. Association of smoking and khat use with HBP among adults in Addis Ababa. 2008; 5(3):1-11. Available at: www.cdc.gov/pcd/issues/2008/jul/07_0137.htm

[23] Muluneh AT, Haileamlak A, Tessema F, Woldemichael K, Asefa M, Mamo Y, et al. Population based survey of chronic non- communicable diseases at Gilgel gibe field research center, Southwest Ethiopia. Ethiop J Health Sci. 2012; 22:161 .

[24] Alemseged F, Haileamlak A, Tegegn A, Tessema F, Woldemichael $\mathrm{K}$, et al. Risk factors for chronic noncommunicable diseases at Gilgel gibe field research center , Southwest Ethiopia :. Ethiop J Health Sci. 2012; 22(1):1-61.

[25] Cortez AL. The international development strategy beyond 2015: Considering demographic dynamics. Economic\& Social Affairs. 2012; 122(1):7-16.

[26] Ibrahim MM, Damasceno A. Hypertension \&pre-hypertension in developing countries. The Lancet; 2012; 380(9841):611-9. Available at: www.thelancet.com
[27] Ferrucci L, Giallauria F and GJ. Epidemiology of Aging. RadiolClin North Am. 2009; 46(4):1-19.

[28] AdugnaA. Non-infectious adult disease.2008:3-5.Available at: www.Ethio-demographyAnd Health. Org

[29] Erem C, Hacihasanoglu A, Kocak M, Deger O, Topbas M. Prevalence of pre-hypertension, hypertension, and associated risk factors among Turkish adults: Trabzon hypertension study. 2008; 31(1):47-58.

[30] Sidhu S, Kumari K. Socio-demographic variables of hypertension among adult Punjabi females. J. Hum. Eco. 2005; $17(3): 211-5$.

[31] Andre Pascal Kengne, Paschal KumAwah, Leopold Fezeuetal. The burden of high blood pressure and related risk factors in Douala, Cameroon. J African Health Sciences. 2007; 7(1):3844.

[32] Tazi MA, Lahmouz F, Arrach ML, Chaouki N. Risk factors for hypertension among. Eastern Mediterranean Health Journal. $2009 ; 15(4): 827-41$.

[33] Sarma VA, Nakarj K. socio-demographic determinant of hypertension. J Hum Ecol, Chitoor District. 2001; 12(3):191-4.

[34] Jf W, Karyabakabo Z, Ndungutse D, Guwatudde D. Prevalence\& factors associated with Hypertension in Rukungiri District, Uganda - A Community-Based Study. J Africa health sci. 2009; 9(3):153-60.

[35] Mayega RW, Makumbi F, Rutebemberwa E, Peterson S. Modifiable socio-behavioural factors associated with overweight and hypertension among persons aged 35 to 60 years in Eastern Uganda. 2012;7(10)

[36] Goma FM, Nzala SH, Babaniyi O, Songolo P, Zyaambo C, Rudatsikira E, et al. Prevalence of hypertension and its correlates in Lusaka urban district of Zambia: a population based survey. Biomed Central Ltd; 2011; 4(1): 1-6. Available at: http://www.intarchmed.com/content/4/1/34

[37] Awoke A, Awoke T, Alemu S, Megabiaw B. Prevalence, and associated factors of hypertension among adults in Gondar, Northwest Ethiopia. 2012; 12 (1):1-6.Available at: http://www.biomedcentral.com/1471-2261/12/113

[38] Giday A, Tadesse B. Prevalence and determinants of hypertension in sidama zone, Ethiopia. Ethiop Med J.2011; 49 (2):139-47.

[39] Rutledge TF. Morbidity and mortality weekly report. Prevalence of selected risk behaviors and chronic diseases and conditions, United States, 2010; 59:2005-8.

[40] Mocumbi, AO. Lack of focus on cardiovascular disease in SSA, Mocumbi. Cardiovascular diagnosis and therapy. Cardiovasculardiagn ther.2012; 2(1):74-77.Available at: http://www.thecdt.org/article/view/103/183

[41] Al-hamdan NA, Al-zalabani AH, Saeed AA. Comparative study of physical activity of hypertensive and normotensives : Saudi Arabia. 2012; 19(3):162-6.

[42] Ali HA, Al-asadi JN. Prevalence and lifestyle determinants of hypertension among secondary school female teachers in Basrah. MJBU. 2009; 27(2):90-94.

[43] Nanchahal K, David W, Wood DA. Alcohol consumption, metabolic cardiovascular risk factors and hypertension in women. 2000:57-64. 
[44] Ramirez SS De, Enquobahrie DA, Nyadzi G, Mjungu D, Magombo F. Prevalence and correlates of hypertension: SSA. Journal of Human Hypertension. 2010; 24(12):786-95. Available at: http://dx.doi.org/10.1038/jhh.2010.14

[45] Tesfaye F, Byass P, Berhane Y, Bonita R. Association of Smoking and Khat ( Catha edulisForsk) Use With HBP Among Adults in Addis Ababa .2008 ; 5(3):1-11.

[46] Chhetri MR. Prevalence and determinants of hypertension and diabetes among elderly population. 2008: 1-214.

[47] A Bener, J Al-Suwaidi, K Al-Jaber, S Al-Marri, IEA Elbagi. Epidemiology of hypertension and its associated risk factors in the Qatari population.Journal of Human Hypertension. Scientific letter. 2004:1-2. Available at: www.nature.com/jhh

[48] Solomon T, Alemseged F. Risk factors for cardiovascular diseases among diabetic patients in Southwest Ethiopia. Ethiop J Health Sci.2008; 20(2):121-128.

[49] Lemma S. Assessment of Risk Factors for Selected Chronic Diseases among Higher Education Students in Addis Ababa. 2007:1-82.
[50] Min H. Social and demographic factors associated with diabetes and hypertension in Hawaii . Hawaii journal of public health. 2009; 3(1):36-41.

[51] Ibrahim NKR, Hijazi NA, Bar AAA. Prevalence \&determinants of pre-hypertension and hypertension among preparatory and secondary school teachers in Jeddah. J Egypt public health assoc. 2008; $83:(3 \& 4): 183-203$.

[52] Getahun1W, GedifT and TesfayeF.Regular Khat (Catha edulis) chewing is associated with elevated diastolic blood pressure among adults in Butajira, Ethiopia: A comparative study. 2010:1-8.

[53] Ravi T, Carlos A; Meir J, Gary C, Eric B.Prospective study of moderate alcohol consumption and risk of hypertension in young women.Arch intern med. 2002; 162:1-6

[54] WHO. World health survey results: Ethiopia 2003. Geneva: WHO. Available at: http://www.who.int/healthinfo/survey/whseth.Ethiopia.Pdf. Accessed on April20, 2013. 